\title{
Editorial
}

\section{Two or three (thousands) things to do before dying}

\author{
P Rovere ${ }^{\star, 1}$ and AA Manfredi ${ }^{1}$
}

1 Immunopathology Unit, Department of Molecular Pathology and Medicine, Cancer Immunotherapy and Gene Therapy Program, Istituto Scientifico H S. Raffaele, via Olgettina 60, 20132 Milano, Italy.

* Corresponding author: P Rovere, Immunopathology Unit, Department of Molecular Pathology and Medicine, Cancer Immunotherapy Program, Istituto Scientifico H S. Raffaele, via Olgettina 60, 20132 Milano, Italy.

E-mail: P.Rovere@hsr.it or A.Manfredi@hsr.it

The decision to die in front of a noxious stimulus, although possibly not easy, looks straightforward. Three reviews published in this issue of Cell Death and Differentiation ${ }^{1-3}$ reveal this simplicity as a mirage. Molecular sensors of cell injury recruit proteolytic activities, which are responsible for the arrest of protein synthesis in dying cells, ${ }^{1}$ for the qualitative modifications of intracellular auto-antigens, including proteins ${ }^{4}$ and nucleic acids ${ }^{2}$ and for the fine regulation of signaling pathways that control cell morphology, cellular repair, cell cycle and RNA splicing. ${ }^{3}$ Cells, and the way they behave in response to injury, dramatically change.

Many modifications mark an irreversible limit: modified cells are condemned. However, after an injury, viable cells survive that underwent partial proteolysis as a consequence of caspase or calpain activation. Such 'undead' cells, which did not complete the apoptotic program, are among us. They may provide clues for biological riddles like T cell anergy, tumor escape and possibly autoimmunity.

Utz and Anderson ${ }^{3}$ focus on a 'sensing' phase which precedes the ultimate commitment to death. During this phase, signaling molecules play a crucial role. The balance among diverse signaling pathways heavily contributes to the eventual cell fate. We are used to consider such balance as the result of the relative level of pro-apoptotic versus anti-apoptotic factors. The transcriptional activation of anti-apoptotic genes, such as IEX-16, directly alters this balance in stressed cells. Regulation occurs also at the level of mRNA splicing, something the authors refer to as 'changing the rules of battle after the whistle blows. ${ }^{3}$ This is the case of the splice variants of the bcl-X gene: cells that express the larger variant $\mathrm{bcl}-\mathrm{X}_{\mathrm{L}}$ have a decreased susceptibility to apoptosis, in contrast to apoptosis prone cells, which express the short variant bcl- $X_{S}$ (see also below).

Several factors cleaved during apoptosis operate at the interface between protection or commitment to apoptosis. Furthermore, each single molecule plays diverse roles, depending on the cell substrate and the concomitant influence of signaling pathways that are activated, deactivated or de-regulated by apoptosis-recruited proteases. Caspases, besides initiator or executioner of the apoptotic program, should be regarded as main determinants of an integrated scenario, in which proteolytic activities regulate the entry into apoptosis as well as cell survival. ${ }^{3}$
This regulation does not obey to a binary logic: the cleavage of signaling molecules results in the re-distribution of crucial components, in the irreversible disruption of their functional activity ${ }^{1,3}$ as well as in the alteration of their substrate specificity. ${ }^{1}$ Caspase-dependent cleavage of kinases may determine their constitutive activation, uncoupling enzymatic sites from regulatory domains. ${ }^{1,3}$ The cleavage of factors involved in cell repair or in cell cycle control will contribute to make survival unlikely. ${ }^{1,3}$ In parallel, caspase-dependent cleave liberates the full proapoptotic activity of other kinases, like MEKK-1, capable in turn to activate other caspases, leading to the amplification of the earliest death signal. ${ }^{3}$ Conversely, survival factors activate the phosphoinositide-3 kinase that via the phosphorylation of selected targets such as BAD and caspase-9 promotes cell survival. ${ }^{1-3}$

Surviving cells will not be the same any more. For example, they will contain partially proteolysed substrates. Anergic $T$ cells, which down-regulated the $T$ cell Receptor $(\mathrm{TcR}) \zeta$ chain, are possible candidate for viable cells bearing in vivo the mark of partial caspase activation. ${ }^{3}$ Limited activation of caspases, although not sufficient for determining $\mathrm{T}$ cell apoptosis, via selective targeting of a crucial component of the TcR signaling complex, may thus contribute to (irreversibly?) skew cell functions in vivo.

The caspase dependent cleavage of the TcR $\zeta$ chain and the ensuing functional paralysis of $T$ cells may help tumors to escape immune recognition. ${ }^{5} \mathrm{~T}$ lymphocytes that recognized via the TcR tumor antigens will not perform antineoplastic activities. The relationship of this pathway with the exploitation of the expression by tumors of ligands for death receptors to control anti-neoplastic responses ${ }^{6}$ is still unexplored. The integration of intracellular signals may be involved in the choice between tumor specific $T$ cell apoptosis and $T$ cell anergy. A crucial influence is possibly played by endogenous (or viral) factors, which control the activation and the functional activities of caspases. ${ }^{7}$

The control of protein synthesis during apoptosis is mediated by the fragmentation or the modification (phosphorylation, association with binding proteins) of initiation factors. The elF4G molecule mediates a core function. This factor bridges protein involved in the association between mRNA and the $40 \mathrm{~S}$ ribosomal subunit. Cleavage of elF4G contributes to the downregulation of protein synthesis in dying cells. Of interest, generated fragments remain associated with ribosome and may still support the initiation of protein synthesis. Clemens and coworkers convincingly suggest that the accumulation of cleavage products in apoptosing cells contribute to perturb the regulation of gene expression at the translational level. ${ }^{1}$ The peculiar characteristics of the enduring function of elF4G fragments, may privilege mRNAs capable of cap-independent translation, leading to qualitative modifications in the proteins the dying cell synthesizes. 
Among them both pro- and anti-apoptotic gene products have been characterized. The balance between the feedback activated by such factors, which include the XIAP inhibitor of apoptosis and the pro-apoptotic Apaf-1 protein, will contribute to the final outcome of the noxious stimulus. ${ }^{1}$

Autoantibodies, i.e. immunoglobulins from patients with systemic autoimmune disease that recognize with high affinity intracellular components, have proved a precious tool for cell biologists. Several cell constituents that elicit continued autoimmunity are cleaved by caspases or granzyme B or otherwise modified during apoptosis. ${ }^{2-4}$ In particular, the U1-snRNP complex is a well-characterized target of autoimmunity and undergoes specific cleavage in apoptosing cells. ${ }^{2}$ This observation is important, since the association of this complex with precursor mRNAs controls their splicing.

Auto-antibody probes allowed the identification of kinases that phosphorylate and control the RNA splicing activity during apoptosis of the SR factors. ${ }^{3}$ Phosphorylated SR factors associate with the U1-snRNP complex during apoptosis and restore the splicing activity of U1-snRNP depleted cell extracts. SR proteins may vicariate components of U1-snRNP that are inactivated during apoptosis. ${ }^{2}$ This event could contribute to switch the control of the splicing of mRNAs coding for molecules that regulate susceptibility to programmed cell death, thus influencing the outcome of a noxious stimulus ${ }^{2}$ and possibly further contributing to sever the intracellular antigen repertoire of dying and viable cells.

Detailed information, excellently reviewed by Degen and co-workers, ${ }^{2}$ is now available on the modifications of other intracellular constituents, like nucleic acids. The degradation of chromosomal DNA, via caspase-dependent cleavage of ICAD (Inhibitor of Caspase Activated DNAse) yields nucleosomes. Nucleosomes represent a key $\mathrm{T}$ and $\mathrm{B}$ cell autoantigen in SLE patients and a striking association exists between the capacity of RNA molecules, including U1-snRNA and Y-RNAs, to be modified by a still unidentified caspase-activated ribonuclease activity and their ability to elicit a continued autoimmune response. A better understanding of the molecular bases of this link, possibly via the recruitment of specialised antigen presenting phagocytes, ${ }^{8,9}$ will be precious for our understanding of the companionship between apoptosis and autoimmunity. ${ }^{10}$

\section{References}

1. Clemens MJ, Bushell M, Jeffrey IW, Pain VM and Morley SJ (2000) Translation initiation factor modifications and the regulation of protein synthesis in apoptotic cells. Cell Death Differ. 7: 603-615

2. Degen WGJ, Pruijn GJM and van Venroij WJ (2000) Caspase dependent cleavage of nucleic acids. Cell Death Differ. 7: 616-627

3. Utz PJ and Anderson P (2000) Life and death decisions: regulation of apoptosis by proteolysis of signaling molecules. Cell Death Differ. 7: 589-602

4. Rosen A and Casciola-Rosen L (1999) Autoantigens as substrates for apoptotic proteases: implications for the pathogenesis of systemic autoimmune disease. Cell Death Differ. 6: 6-12

5. Whiteside TL (1999) Signaling defects in T lymphocytes of patients with malignancy. Cancer Immunol. Immunother. 48: 346-352

6. O'Connell J, Bennett MW, O'Sullivan GC, Collins JK and Shanahan F (1999) The Fas counterattack: cancer as a site of immune privilege. Immunology Today 20 : $46-52$

7. Ekert PG, Silke J and Vaux DL (1999) Caspase inhibitors. Cell Death Differ. 6 : $1081-1086$

8. Rovere P, Vallinoto C, Bondanza A, Crosti MC, Rescigno M, RicciardiCastagnoli P, Rugarli C and Manfredi AA (1998) Bystander apoptosis triggers dendritic cell maturation and antigen presenting function. J. Immunol. 161: 4467-4471

9. Albert ML, Sauter B and Bhardway N (1998) Dendritic cells acquire antigen from apoptotic cells and induce class I-restricted CTLs. Nature 392: 86-89

10. Clemens MJ, van Venrooij WJ and van de Putte LBA (2000) Apoptosis and autoimmunity. Cell Death Differ. 7: 131-133 